\title{
The application of a new formula for measuring the health status for all from the mathematical point of view.
}

\author{
Ali Mehrabi Tavana
}

\begin{abstract}
Health had a qualitative definition and it has always been said that health is a complete physical, mental, social and spiritual, well-being. The purposes of this study were present and determine a new formula for measuring the health status from the mathematical point of view. Methods: The selfdeveloped formula in this study was evaluated in few cases and evaluated experimentally and software SPSS VERSION 19.0 was used for statistical analysis. Results: A few examples of with different health conditions have been calculated in this formula. Based on my formula which was applied in these examples, each health scores were measured 40.05, 42.20, 44.10 and 46.00 respectively. Discussion; Calculated numbers imply the health status of a person in terms of its specific characteristics, which is calculated accordingly. Thus, these findings showed that the formula which was suggested by us will be able to measure the health of individuals at any level in the World.
\end{abstract}

Keywords - application; formula; health; mathematical point.

\section{INTRODUCTION}

In international health organizations, health is mostly defined qualitatively since its inception; the World Health Organization has only addressed the definition of quality of life. They said that health is a complete physical and social well-being and encourages people to have it. Of course, in recent years; spiritual health has also been added to this definition [1]. Today, communicable and non-communicable diseases threaten people's health, people are more or less aware of their impact on their health, but do not know how much these diseases decrease their health in terms of their health in addition, does this decrease significantly related to their health from temporary or permanent illnesses or not and whether they are basically themselves and their healthcare professionals aware of how much this impact is and how they can overcome it. Helping to promote the health of the individual and his community, and basically understanding how each person's health can increase the health of the individual and society.

Numerous examples for health are brought. At the same time, note that this formula can lead to changes in human health. Just put my formula (Professor Mehrabi Tavana) in front of you and enter the health status of your family or community, and then get the real and calculated number. Of course, this is the beginning of what you will be aware of a little about your health. After that, you or your health or health authorities have thought about how to improve your health. Of course, you should note that the best health in this formula is 100 . Everyone should try to work towards this number. Therefore, once my formula is calculated based on the health of the people of the world, after which the governments will understand how well the health of each community is and how it is distributed in the city and the countryside. How is today the next year and the following years, how much should be spent on health to reach 100 for all people, how much to spend on them in cities and how much to spend in the countryside, all from a mathematically perceptible point of view. Thus, everyone will understand the importance of using this mathematical formula to measure health and use it in her/his own health and society and make sure that the world will know its health and will see its progress. The purposes of this study were present and determine a new formula for measuring the health status from the mathematical point of view.

\section{II.METHOD}

The methodology of my own innovative formula has been tested in this study and examples of different people with different health conditions have been calculated in this formula (Box 1). Based on the formula which was introduced by me different examples will be calculated. The data which were used in these examples are hypothetically.

\section{Box1. Formula for calculation of human health which was created by Professor Ali Mehrabi Tavana and his son Mehdi Mehrabi Tavana 2017.}

\section{$\mathrm{HU} / \mathbf{1 0 0}=\mathrm{P} / \mathbf{1 0 0}+\mathrm{M} / \mathbf{1 0 0}+\mathrm{SO} / \mathbf{1 0 0}+\mathrm{SP} / \mathbf{1 0 0}$}

$\mathrm{HU} / 100=(\mathrm{P}=\mathrm{p} 1+\mathrm{p} 2+\mathrm{p} 3+\mathrm{p} 4+\mathrm{p} 5+\mathrm{p} 6+\mathrm{p} 7+\mathrm{p} 8+\mathrm{p} 9+\mathrm{p} 10+\mathrm{p} 11 / 100)$ $+(\mathrm{M}=\mathrm{m} 1+\mathrm{m} 2+\mathrm{m} 3+\mathrm{m} 4+\mathrm{m} 5+\mathrm{m} 6+\mathrm{m} 7+\mathrm{m} 8+\mathrm{m} 9+\mathrm{m} 10+\mathrm{m} 11 / 100)$ $+(\mathrm{SO}=\mathrm{so} 1+\mathrm{so} 2+\mathrm{so} 3+\mathrm{so} 4+\mathrm{so} 5+\mathrm{so} 6+\mathrm{so} 7+\mathrm{so} 8+\mathrm{so} 9+\mathrm{so} 10+\mathrm{so} 11+$ $\mathrm{so} 12+\mathrm{so} 13+\mathrm{so} 14 / 100)+(\mathrm{SP}=\mathrm{sp} 1+\mathrm{sp} 2+\mathrm{sp} 3+\mathrm{sp} 4+\mathrm{sp} 5+\mathrm{sp} 6+\mathrm{sp} 7+\mathrm{sp} 8$ + sp9+sp10+sp11+sp12+sp13)/100.

Keys: My formula for calculation of human health: $\mathrm{Huh} / 100=\mathrm{P} / 100+\mathrm{M} / 100+\mathrm{SO} / 100+\mathrm{SP} / 100$.

Firstly, different physical health elements (i.e. $\mathrm{P}=\mathrm{np}$;p0; age, $\mathrm{p} 1=$ genetic heritance, $\mathrm{p} 2=$ skeleton, $\mathrm{p} 3=$ normal heart function , $\mathrm{p} 4=$ normal lung function, $\mathrm{p} 5=$ normal brain function, $\mathrm{p} 6=$ normal , GI function, $\mathrm{p} 7=$ normal skin (without skin disease), $\mathrm{p} 8=$ normal $\mathrm{ENT}, \mathrm{p} 9=$ normal eye vision, p10=normal Genitourinary Tract ,p11=normal teeth, $\mathrm{p} 12=$ fertility .

$\mathrm{P}=\mathrm{p} 0+\mathrm{p} 1+\mathrm{p} 2+\mathrm{p} 3+\mathrm{p} 4+\mathrm{p} 5+\mathrm{p} 6+\mathrm{p} 7+\mathrm{p} 8+\mathrm{p} 9+\mathrm{p} 10+\mathrm{p} 11+\mathrm{p} 12+\ldots /$ 100.

Secondly different mental health elements (i.e., $\mathrm{M}=\mathrm{nm}$;m0: mental health score in the society, $\mathrm{ml}=$ genetic heritance, $\mathrm{m} 2=$ normal mentality, $\mathrm{m} 3=$ normal behavior, $\mathrm{m} 4=$ =normal sleeping, $\mathrm{m} 5=$ normal communication , $\mathrm{m} 6=$ not aggressive, $\mathrm{m} 7$ =happiness, $\mathrm{m} 8=$ =family relationship ,p9=friend's relationship, $\mathrm{m} 10=$ independency, $\mathrm{m} 11=$ self-motivation) $\mathrm{M}=\mathrm{m} 0=\mathrm{m} 1+\mathrm{m} 2+\mathrm{m} 3+\mathrm{m} 4+\mathrm{m} 5+\mathrm{m} 6+\mathrm{m} 7+\mathrm{m} 8+\mathrm{m} 9+\mathrm{m} 10+\mathrm{m} 11 /$ 100. Thirdly social health elements $(\mathrm{SO}=\mathrm{nso}$; so0: social 
health score in the society, So1=job, So2=life style, So3=income, So4=social support, So5=insurance, So6=nonsmoker, So7=literacy, So8=social activity, So9=access to healthy foods, water and housing, So10=marriage, So 11=life Expectancy, So12=low incidence of crime, So13=road accident mortality rate, So14=national health score $(\ldots .+\mathrm{SO}=\mathrm{so} 0+\mathrm{so} 1+\mathrm{so} 2+\mathrm{so} 3+$ so $4+$ so $5+$ so $6+$ so $7+$ so8+so9+so10+so11+so12+so13+so14+.../100.

Fourthly spiritual health elements $(\mathrm{SP}=\mathrm{nsp}$; spiritual health score in the society, $\mathrm{Sp} 1=$ belief in God, $\mathrm{Sp} 2=$ Continuous worship (continuous practice of faith), Sp3=Satisfaction pleasing to God, Sp4=Self-content ,Sp5=humanitarian activities, Sp6=other people's consent $\mathrm{Sp} 7=$ daily best practice, $\mathrm{Sp} 8=$ stress level, Sp9=Belief in the Day of Judgment, Sp10=dignity, Sp 11=Loving others, S12=Trust in God, $\mathrm{Sp} 13=$ patience. .... $+\mathrm{SP}=\mathrm{sp} 0+\mathrm{sp} 1+\mathrm{sp} 2+\mathrm{sp} 3+\mathrm{sp} 4+$ sp5+ sp6+ sp7+sp8+sp9+sp10+sp11+sp12+sp13/100.

\section{III.RESULTS:}

\section{Example 1:}

A man from Africa is 25 years old $(\mathrm{P} 0=100)$ and has no genetic heritance disorder $(\mathrm{p} 1=100)$, Normal skeleton ( $\mathrm{p} 2=100)$, normal heart function $(\mathrm{p} 3=100)$, normal lung function $(\mathrm{p} 4=100)$. Normal brain function $(\mathrm{p} 5=100)$ normal GI function ( $\mathrm{p} 6=100)$ with abnormal skin disease with cutaneous leishmaniasis) ( $\mathrm{p} 7=50$ normal ENT $(\mathrm{p} 8=100)$ abnormal eye vision $(\mathrm{p} 9=50)$. Normal Genitourinary Tract $(\mathrm{p} 10=100)$ and normal teeth $(\mathrm{p} 11=100)$. His physical health could be calculated as follows:

\section{$P=100+100+100+100+100+100+100+50+100+50+100+10$ $\mathbf{0} / \mathbf{1 0 0}=\mathbf{1 1 . 0}$}

Such a man ( 25 years old) $\mathrm{M} 0=100$ has got the following mental health criteria, Not genetic heritance disorder $\mathrm{m} 1=100$, normal mentality $\mathrm{m} 2=100$. Normal behavior $\mathrm{m} 3=100$. Very abnormal sleeping $\mathrm{m} 4=25)$, normal communication $(\mathrm{m} 5=100)$, not aggressive $(\mathrm{m} 6=100)$ very good happiness $(\mathrm{m} 7=100)$, excellent family relationship $(\mathrm{m} 8=100)$, normal friend's relationship $(\mathrm{p} 9=100)$ Independency $(\mathrm{m} 10=80)$ and good self-motivation $(\mathrm{m} 11=75)$ Thus, his mental health could be calculated as the follows' $=100+100+100+100+25+100+100+100+100+100+$ $80+75 / 100=10.80$

With regard to his social health the following elements could be considered $\mathrm{So} 0=50$, job, (jobless) So1=0, life style, So $2=60$, income $\mathrm{So} 3=0$, social support So4 $=0$, insurance So5 $=100$, not smoker (heavy smoker) So6 $=0$, literacy (primary school graduate $\mathrm{So} 7=25$ ), social activity (intermediate) So8 $=50$, access to safe foods, water and housing (medium) So9=50, married So10=100, life expectancy So11 $=40$, low incidence of crime So12=75, mortality rate of road accident (high) So13=25 health nationality score (weak) So14 $=75$.

$\mathrm{SO}=50+0+60+0+0+100+0+25+50+50+100+40+75+25+25 /$

\section{0}

$600 / 100=6.0$

Finally, about his SPIRITUAL condition, the following elements were considered:

Sp0:100, Belief in God Sp1=100, Continuous worship $\mathrm{Sp} 2=100$ Satisfaction pleasing to God Sp3 $=100$, Self-content Sp4=100 humanitarian activities $\mathrm{Sp} 5=50$, Consent of the people $\mathrm{Sp} 6=75$ daily best practice $\mathrm{Sp} 7=50$, stress level (low) Sp8 $=50$, Belief in the Day of Judgment Sp9=100, Dignity Sp10 $=100$, Loving Others Sp 11=100, Trust in God S12=100, patience $\mathrm{Sp} 13=100$

$\mathrm{SP}=100+100+100+100+100+50+75+50+50+100+100+100$ $+100+100 / 100$.

\section{$1225 / 100=12.25$}

HU/100 $=11.0+10.80+6.0+12.25=40.05$

This means the man has received a score of $\mathbf{4 0 . 0 5}$ out of 100 anyway (Table1).

\begin{tabular}{|c|c|c|c|c|c|c|c|}
\hline $\begin{array}{l}\text { Phys } \\
\text { ical } \\
\text { Fact } \\
\text { ors }\end{array}$ & $\begin{array}{l}\text { val } \\
\text { ues }\end{array}$ & $\begin{array}{l}\text { Men } \\
\text { tal } \\
\text { Fact } \\
\text { ors }\end{array}$ & $\begin{array}{l}\text { val } \\
\text { ues }\end{array}$ & $\begin{array}{l}\text { Soci } \\
\text { al } \\
\text { Fact } \\
\text { ors }\end{array}$ & $\begin{array}{l}\text { val } \\
\text { ues }\end{array}$ & $\begin{array}{l}\text { Spiri } \\
\text { tual } \\
\text { Facto } \\
\text { rs }\end{array}$ & $\begin{array}{l}\text { val } \\
\text { ues }\end{array}$ \\
\hline p0 & 100 & $\mathrm{~m} 0$ & 100 & so0 & 50 & sp0 & 100 \\
\hline p1 & 100 & $\mathrm{~m} 1$ & 100 & sol & 0 & sp1 & 100 \\
\hline p2 & 100 & $\mathrm{~m} 2$ & 100 & so2 & 60 & $\mathrm{sp} 2$ & 100 \\
\hline p3 & 100 & $\mathrm{~m} 3$ & 100 & so3 & 0 & sp3 & 100 \\
\hline $\mathrm{p} 4$ & 100 & $\mathrm{~m} 4$ & 25 & so 4 & 0 & $\mathrm{sp} 4$ & 100 \\
\hline p5 & 100 & $\mathrm{~m} 5$ & 100 & so 5 & 100 & $\mathrm{sp} 5$ & 50 \\
\hline p6 & 100 & m6 & 100 & so6 & 0 & sp6 & 75 \\
\hline p7 & 50 & $\mathrm{~m} 7$ & 100 & so 7 & 25 & sp7 & 50 \\
\hline p8 & 100 & $\mathrm{~m} 8$ & 100 & so8 & 50 & sp8 & 50 \\
\hline p9 & 50 & $\mathrm{~m} 9$ & 100 & so9 & 50 & sp9 & 100 \\
\hline $\mathrm{p} 10$ & 100 & $\mathrm{~m} 10$ & 80 & so10 & 100 & sp10 & 100 \\
\hline \multirow[t]{4}{*}{ p11 } & 100 & $\mathrm{~m} 11$ & 75 & sol1 & 40 & sp11 & 100 \\
\hline & & & & so12 & 75 & sp12 & 100 \\
\hline & & & & so13 & 25 & sp13 & 100 \\
\hline & & & & so14 & 25 & & \\
\hline $\begin{array}{c}\text { Mean } \\
/ 100\end{array}$ & $\begin{array}{l}11 . \\
0\end{array}$ & & $\begin{array}{l}10 . \\
80\end{array}$ & & 6.0 & & $\begin{array}{l}12 . \\
25\end{array}$ \\
\hline $\begin{array}{c}=40 . \\
05\end{array}$ & & & & & & & \\
\hline
\end{tabular}

\section{Example 2:}

A woman from Africa is 40 years old $(\mathrm{P} 0=100)$ and has no genetic heritance disorder $(\mathrm{p} 1=100)$, Normal skeleton (p2=100), normal heart function ( $\mathrm{p} 3=100)$, abnormal lung function $(\mathrm{p} 4=50)$. Normal brain function $(\mathrm{p} 5=100)$ normal GI function $(\mathrm{p} 6=100)$ with normal skin $\mathrm{p} 7=100$ normal ENT $(\mathrm{p} 8=100)$ normal eye vision ( $\mathrm{p} 9=100)$. Normal Genitourinary Tract $(\mathrm{p} 10=100)$ and normal teeth $(\mathrm{p} 11=100)$.

His physical health could be calculated as follows: $\mathrm{P}=100+100+100+100+50+100+100+100+100+100+100+$ $100 / 100=11.50$.

Such a women $\mathrm{m} 0=100$ has got the following mental health criteria, Not genetic heritance disorder $\mathrm{ml}=100$, normal mentality $\mathrm{m} 2=100$. Normal behavior $\mathrm{m} 3=100$. Very abnormal sleeping $\mathrm{m} 4=25$ ), normal communication $(\mathrm{m} 5=100)$, not aggressive $\mathrm{m} 6=100)$ very good happiness $(\mathrm{m} 7=100)$, excellent family relationship $(\mathrm{m} 8=100)$, normal friend's relationship $(\mathrm{p} 9=100)$ Independency $(\mathrm{m} 10=50)$ and very good self-motivation $(\mathrm{m} 11=100)$ Thus, his mental health could be calculated as the follows:

\section{$M=100+100+100+100+25+100+100+100+100+100+50+$ $100 / 100=10.75$}

With regard to her social health $(\mathrm{So} 0=50)$ the following elements could be considered job, (jobless) Sol=0, life style, 
So $2=50$, income So3 $=0$, social support So4 $=0$, insurance So5 $=100$, not smoker (no smoker) So6 $=100$, literacy (primary school graduate So7 $=25$ ), social activity (intermediate) So8 $=50$, access to safe foods, water and housing (medium) So9 $=50$, married So10=100, life expectancy So11 $=50$, low incidence of crime So12 $=75$, mortality rate of road accident (high) So13=25 health nationality score (weak) So $14=25$.

\section{$\mathrm{SO}=\mathbf{5 0}+0+50+0+0+100+100+25+50+50+100+50+75+25+$}

\section{5/100}

$700 / 100=7.0$

Finally, about her SPIRITUAL condition, the following elements were considered:

Sp0:100, Belief in God Sp1=100, Continuous worship $\mathrm{Sp} 2=100$ Satisfaction pleasing to God Sp3 $=100$, Self-content Sp4=100 humanitarian activities Sp5=100, Consent of the people $\mathrm{Sp} 6=100$ daily best practice $\mathrm{Sp} 7=50$, stress level (low) $\mathrm{Sp} 8=50$, Belief in the Day of Judgment Sp9=100, Dignity Sp10=100, Loving Others Sp 11=100, Trust in God S12=100, patience $\mathrm{Sp} 13=100$

$\mathrm{SP}=100+100+100+100+100+100+75+50+50+100+100+10$ 0+100+100/100.

\section{$1275 / 100=12.75$}

\section{HU/100 $=11.50+10.75+7.0+12.75=42.20$}

This means the women has received a score of $\mathbf{4 2 . 2 0}$ out of 100 anyway (Table2).

Table 2. The health of person in example 2 with different health aspects and their relevant factors.

\begin{tabular}{|c|c|c|c|c|c|c|c|}
\hline $\begin{array}{l}\text { Phys } \\
\text { ical } \\
\text { Fact } \\
\text { ors }\end{array}$ & $\begin{array}{l}\text { val } \\
\text { ues }\end{array}$ & $\begin{array}{c}\text { Men } \\
\text { tal } \\
\text { Fact } \\
\text { ors }\end{array}$ & $\begin{array}{l}\text { val } \\
\text { ues }\end{array}$ & $\begin{array}{c}\text { Soci } \\
\text { al } \\
\text { Fact } \\
\text { ors }\end{array}$ & $\begin{array}{l}\text { val } \\
\text { ues }\end{array}$ & $\begin{array}{c}\text { Spiri } \\
\text { tual } \\
\text { Facto } \\
\text { rs }\end{array}$ & $\begin{array}{l}\text { val } \\
\text { ues }\end{array}$ \\
\hline $\mathrm{p} 0$ & 100 & $\mathrm{~m} 0$ & 100 & so0 & 50 & sp0 & 100 \\
\hline p1 & 100 & $\mathrm{~m} 1$ & 100 & sol & 0 & sp1 & 100 \\
\hline p2 & 100 & $\mathrm{~m} 2$ & 100 & so2 & 50 & $\mathrm{sp} 2$ & 100 \\
\hline p3 & 100 & $\mathrm{~m} 3$ & 100 & so3 & 0 & sp3 & 100 \\
\hline $\mathrm{p} 4$ & 50 & $\mathrm{~m} 4$ & 25 & so4 & 0 & sp4 & 100 \\
\hline p5 & 100 & $\mathrm{~m} 5$ & 100 & so5 & 100 & sp5 & 100 \\
\hline p6 & 100 & $\mathrm{~m} 6$ & 100 & so6 & 100 & sp6 & 75 \\
\hline p7 & 100 & $\mathrm{~m} 7$ & 100 & so 7 & 25 & sp7 & 50 \\
\hline p8 & 100 & $\mathrm{~m} 8$ & 100 & so8 & 50 & sp8 & 50 \\
\hline p9 & 100 & $\mathrm{~m} 9$ & 100 & so9 & 50 & sp9 & 100 \\
\hline p10 & 100 & $\mathrm{~m} 10$ & 50 & so10 & 100 & sp10 & 100 \\
\hline \multirow[t]{4}{*}{ p11 } & 100 & $\mathrm{~m} 11$ & 100 & so11 & 50 & sp11 & 100 \\
\hline & & & & so 12 & 75 & sp12 & 100 \\
\hline & & & & so13 & 25 & sp13 & 100 \\
\hline & & & & so14 & 25 & & \\
\hline Mean & 11. & & 10. & & 7.0 & & 12. \\
\hline$/ 100$ & 50 & & 75 & & & & 75 \\
\hline $\begin{array}{c}=42 . \\
20\end{array}$ & & & & & & & \\
\hline
\end{tabular}

\section{Example 3:}

A child from Europe is 10 -year-old $\mathrm{p} 0=100$ and has no genetic heritance disorder $(\mathrm{p} 1=100)$, Normal skeleton ( $\mathrm{p} 2=100)$, normal heart function $(\mathrm{p} 3=100)$, normal lung function (p4=100). Normal brain function $(\mathrm{p} 5=100)$ normal GI function ( $\mathrm{p} 6=100)$ with normal skin) ( $\mathrm{p} 7=100$ normal ENT $(p 8=100)$ normal eye vision $(p 9=100)$. Normal Genitourinary Tract $(\mathrm{p} 10=100)$ and normal teeth $(\mathrm{p}=100)$.

His physical health could be calculated as follows:

$P=100+100+100+100+100+100+100+100+100+100+100+$ $100 / 100=12.0$

Such a child has got the following mental health criteria $\mathrm{p} 0=100$, Not genetic heritance disorder $\mathrm{ml}=100$, normal mentality $\mathrm{m} 2=100$. Normal behavior $\mathrm{m} 3=100$. Very abnormal sleeping $\mathrm{m} 4=25$ ), normal communication $(\mathrm{m} 5=100)$, not aggressive $\mathrm{m} 6=100)$ happiness (crying all the time $(\mathrm{m} 7=25)$, excellent family relationship $(\mathrm{m} 8=100)$, normal friend's relationship $(\mathrm{p} 9=100)$ Independency $(\mathrm{m} 10=100)$ and good self-motivation $(\mathrm{m} 11=100)$ Thus, his mental health could be calculated as the follows:

$M=100+100+100+100+25+100+100+25+100+100+100+10$ $\mathbf{0} / \mathbf{1 0 0}=\mathbf{1 0 . 5 0}$

With regard to his/her social health $(\mathrm{SO} 0=100)$ the following elements could be considered job, (jobless) Sol $=0$, life style, So $2=60$, income So3 $=0$, social support So4 $=100$, insurance So5 $=100$, not smoker (no smoker) So6 $=100$, literacy (primary school So7 $=25$ ), social activity (intermediate) So8 $=50$, access to safe foods, water and housing (excellent) So9 $=100$, married So $10=0$, life expectancy Sol1 $=50$, incidence of crime So12 $=100$, mortality rate of road accident (very low) So $13=75$ health nationality score (excellent) So14 $=100$.

$\mathrm{SO}=100+0+60+0+100+100+100+25+50+100+0+50+100+7$ $\mathbf{5}+\mathbf{2 5} / \mathbf{1 0 0}$

$935 / 100=9.35$

Finally, about his/her SPIRITUAL condition, the following elements were considered:

Sp0:100, Belief in God Sp1 $=100$, Continuous worship $\mathrm{Sp} 2=100$ Satisfaction pleasing to God Sp3 $=100$, Self-content Sp4=100 humanitarian activities $\mathrm{Sp} 5=50$, Consent of the people $\mathrm{Sp} 6=75$ daily best practice $\mathrm{Sp} 7=50$, stress level (low) $\mathrm{Sp} 8=50$, Belief in the Day of Judgment Sp9=100, Dignity Sp10 $=100$, Loving Others Sp 11=100, Trust in God S12=100, patience $\mathrm{Sp} 13=100$

$\mathrm{SP}=100+100+100+100+100+50+75+50+50+100+100+100$

$+100+100 / 100$.

$1225 / 100=12.25$

$\mathrm{HU} / \mathbf{1 0 0}=\mathbf{1 2 . 0}+\mathbf{1 0 . 5 0}+\mathbf{9 . 3 5}+\mathbf{1 2 . 2 5}=\mathbf{4 4 . 1 0}$

This means the child has received a score of $\mathbf{4 4 . 1 0}$ out of 100 anyway (Table3).
Table 3 . The health of person in example 1 with different health aspects and their relevant factors.

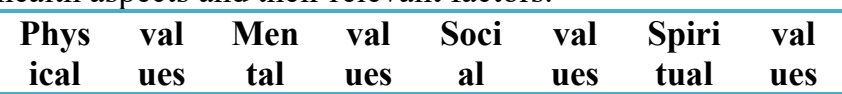




\begin{tabular}{|c|c|c|c|c|c|c|c|}
\hline $\begin{array}{c}\text { Fact } \\
\text { ors }\end{array}$ & & $\begin{array}{l}\text { Fact } \\
\text { ors }\end{array}$ & & $\begin{array}{c}\text { Fact } \\
\text { ors }\end{array}$ & & $\begin{array}{c}\text { Facto } \\
\text { rs }\end{array}$ & \\
\hline p0 & 100 & $\mathrm{~m} 0$ & 100 & so0 & 100 & sp0 & 100 \\
\hline p1 & 100 & $\mathrm{~m} 1$ & 100 & sol & 0 & sp1 & 100 \\
\hline p2 & 100 & $\mathrm{~m} 2$ & 100 & so2 & 60 & sp2 & 100 \\
\hline p3 & 100 & $\mathrm{~m} 3$ & 100 & so3 & 0 & $\mathrm{sp} 3$ & 100 \\
\hline p4 & 100 & $\mathrm{~m} 4$ & 25 & so4 & 100 & sp4 & 100 \\
\hline p5 & 100 & $\mathrm{~m} 5$ & 100 & so5 & 100 & $\mathrm{sp} 5$ & 50 \\
\hline p6 & 100 & m6 & 100 & so6 & 100 & sp6 & 75 \\
\hline p7 & 100 & $\mathrm{~m} 7$ & 25 & so 7 & 25 & sp7 & 50 \\
\hline p8 & 100 & $\mathrm{~m} 8$ & 100 & so8 & 50 & sp8 & 50 \\
\hline p9 & 100 & m9 & 100 & so 9 & 100 & sp9 & 100 \\
\hline p10 & 100 & $\mathrm{~m} 10$ & 100 & so 10 & 0 & sp10 & 100 \\
\hline \multirow[t]{4}{*}{ p11 } & 100 & $\mathrm{~m} 11$ & 100 & sol1 & 50 & sp11 & 100 \\
\hline & & & & so12 & 100 & sp12 & 100 \\
\hline & & & & so13 & 75 & sp13 & 100 \\
\hline & & & & sol4 & 25 & & \\
\hline Mean & 12. & & 10. & & 9.3 & & 12. \\
\hline$/ 100$ & 0 & & 50 & & 5 & & 25 \\
\hline $\begin{array}{c}=44 . \\
10\end{array}$ & & & & & & & \\
\hline
\end{tabular}

\section{Example 4:}

You suppose that become older after 80 years his/her health score could be changed.

$\mathrm{P} 0=25$, and has no genetic heritance disorder $(\mathrm{p} 1=100)$, Normal skeleton $(\mathrm{p} 2=100)$, normal heart function $(\mathrm{p} 3=100)$, normal lung function $(\mathrm{p} 4=100)$. Normal brain function $(\mathrm{p} 5=100)$ normal GI function $(\mathrm{p} 6=75)$ with normal skin) ( $\mathrm{p} 7=100$ abnormal ENT (p8=75) abnormal eye vision (p9=75). Normal Genitourinary Tract $(\mathrm{p} 10=100)$ and abnormal teeth $(\mathrm{p}=25)$.

His physical health could be calculated as follows:

$P=25+100+100+100+100+100+75+100+75+75+100+25 / 10$ $0=9.75$

Such that man/women $\mathrm{m} 0=100$ has got the following mental health criteria, Not genetic heritance disorder $\mathrm{ml}=100$, normal mentality $\mathrm{m} 2=100$. Normal behavior $\mathrm{m} 3=100$. Very abnormal sleeping $\mathrm{m} 4=25)$, normal communication $(\mathrm{m} 5=100)$, not aggressive $\mathrm{m} 6=100)$ happiness $(\mathrm{m} 7=100)$, excellent family relationship $(\mathrm{m} 8=100)$, normal friend's relationship $(\mathrm{p} 9=100)$ Independency $(\mathrm{m} \mathrm{10}=100)$ and good self-motivation $(\mathrm{m} 11=100)$.

Thus, his mental health could be calculated as the follows:

$M=100+100+100+100+25+100+100+100+100+100+100+$

\section{$100 / 100=11.25$}

With regard to his/her social health $(\mathrm{So} 0=100)$ the following elements could be considered job, (employment ) So1=100, life style, So2 $=100$, income So3 $=100$, social support So $4=100$, insurance So5 $=100$, not smoker (heavy smoker) So6 $=0$, literacy (B.Sc. So7=75), social activity (intermediate) So8 $=50$, access to safe foods, water and housing (excellent) So9=100, married So10=100, life expectancy So11 $=100$, incidence of crime So12=100, mortality rate of road accident (very low) So13=75 health nationality score (excellent) So14 $=100$.

$\mathrm{SO}=100+100+100+100+100+100+0+75+50+100+100+100$ $+\mathbf{1 0 0}+\mathbf{7 5}+\mathbf{1 0 0} / \mathbf{1 0 0}$

$1300 / 100=13.0$

Finally, about his/her SPIRITUAL condition, the following elements were considered:
$\mathrm{Sp} 0=50$, Belief in God $\mathrm{Sp} 1=100$, Continuous worship $\mathrm{Sp} 2=100$ Satisfaction pleasing to God Sp3 $=100$, Self-content $\mathrm{Sp} 4=100$ humanitarian activities $\mathrm{Sp} 5=100$, Consent of the people $\mathrm{Sp} 6=75$ daily best practice $\mathrm{Sp} 7=100$, stress level (high) $\mathrm{Sp} 8=25$, Belief in the Day of Judgment Sp9=100, Dignity Sp10=100, Loving Others Sp 11=100, Trust in God $\mathrm{S} 12=100$, patience $\mathrm{Sp} 13=100$

$\mathrm{SP}=\mathbf{5 0}+\mathbf{1 0 0}+\mathbf{1 0 0}+\mathbf{1 0 0}+\mathbf{1 0 0}+\mathbf{1 0 0}+\mathbf{7 5}+\mathbf{5 0}+\mathbf{2 5}+\mathbf{1 0 0}+\mathbf{1 0 0}+100$ $+100+100 / 100$.

\section{$1200 / 100=12.00$}

HU/100 $=9.75+11.25+13+12.00=46.00$

This means the man/woman has received a score of $\mathbf{4 6 . 0 0}$ out of 100 anyway (Table4).

Table 4 . The health of person in example 4 with different health aspects and their relevant factors.

\begin{tabular}{|c|c|c|c|c|c|c|c|}
\hline $\begin{array}{l}\text { Phys } \\
\text { ical } \\
\text { Fact } \\
\text { ors }\end{array}$ & $\begin{array}{l}\text { val } \\
\text { ues }\end{array}$ & $\begin{array}{l}\text { Men } \\
\text { tal } \\
\text { Fact } \\
\text { ors }\end{array}$ & $\begin{array}{l}\text { val } \\
\text { ues }\end{array}$ & $\begin{array}{l}\text { Soci } \\
\text { al } \\
\text { Fact } \\
\text { ors }\end{array}$ & $\begin{array}{l}\text { val } \\
\text { ues }\end{array}$ & $\begin{array}{l}\text { Spiri } \\
\text { tual } \\
\text { Facto } \\
\text { rs }\end{array}$ & $\begin{array}{l}\text { Val } \\
\text { ues }\end{array}$ \\
\hline $\mathrm{p} 0$ & 25 & $\mathrm{~m} 0$ & 100 & so0 & 100 & sp0 & 50 \\
\hline p1 & 100 & $\mathrm{~m} 1$ & 100 & sol & 100 & sp1 & 100 \\
\hline p2 & 100 & $\mathrm{~m} 2$ & 100 & so 2 & 100 & $\mathrm{sp} 2$ & 100 \\
\hline p3 & 100 & $\mathrm{~m} 3$ & 100 & so3 & 100 & sp3 & 100 \\
\hline $\mathrm{p} 4$ & 100 & $\mathrm{~m} 4$ & 25 & so4 & 100 & sp4 & 100 \\
\hline p5 & 100 & $\mathrm{~m} 5$ & 100 & so5 & 100 & $\mathrm{sp} 5$ & 100 \\
\hline p6 & 75 & $\mathrm{~m} 6$ & 100 & so6 & 0 & sp6 & 75 \\
\hline p7 & 100 & $\mathrm{~m} 7$ & 100 & so 7 & 75 & sp7 & 50 \\
\hline p8 & 75 & $\mathrm{~m} 8$ & 100 & so8 & 50 & sp8 & 25 \\
\hline p9 & 75 & $\mathrm{~m} 9$ & 100 & so9 & 100 & sp9 & 100 \\
\hline p10 & 100 & $\mathrm{~m} 10$ & 100 & so 10 & 100 & sp10 & 100 \\
\hline \multirow[t]{4}{*}{ p11 } & 25 & $\mathrm{~m} 11$ & 100 & sol1 & 100 & sp11 & 100 \\
\hline & & & & so12 & 100 & sp12 & 100 \\
\hline & & & & so13 & 75 & sp13 & 100 \\
\hline & & & & so14 & 100 & & \\
\hline Mea & 9.7 & & 11. & & 13. & & 12.0 \\
\hline $\begin{array}{c}\mathrm{n} / \\
100\end{array}$ & 5 & & 25 & & 0 & & \\
\hline $\begin{array}{c}=46 . \\
00\end{array}$ & & & & & & & \\
\hline
\end{tabular}

\section{Discussion:}

These are the advantages of formula which I have created thus the diseases could be added or omitted based on sex, age or regions or even based on weight of the diseases for example very chronic diseases with their high mortality rate and person with disability are also could be considered in this present formula. Weight of the diseases (untreated or treated) has specific value which its value could be added in formula too. The results of each example vary from one to another because the physical, psychological, social, and spiritual status of each individual is completely different, and this difference is included in the formula with different numbers in the formula and is calculated for each example of numbers, which expresses in seven examples which I have submitted, each health scores were 40.05, 42.20, 44.10 and 46.00 respectively. The data for above example are summarized in Table 1-4. The first the formula is well designed and, secondly, it can be calculated in the samples of the community in different people with a different health status. 
Therefore, we are the ones that we can set quantitative criteria in the health formula and calculate it. Of course, these numbers will change with age, sex, location, or diseases, or even untreated or cure the disease, and will show a person's health at a range of $0-100$ totally or for each health section separately. We can bring several examples of the population of the world and calculate their health scores. It is important that this formula has the ability to assess each individual according to different physical, mental, social, and spiritual health conditions and give his/her health number and ask them to constantly strive for his /her health [2]. Otherwise, health rating may decrease, and this health rating can be exploited for his and her health authorities for recruitment and insurance policy. The good and excellent auctions pay the minimum insurance and the premium should pay more. You could put different criteria in different subclass of physical, mental, social, and spiritual health, and you could consider different range of score. Of course, this amount is a contract and can change. It is possible to calculate the number of health factors which can be introduced by specialists for different health sections including physical, mental, social and spiritual. In addition, each section which is mentioned earlier could be subdivided with a numbers that agreed among specialist which are more relevant in being healthy. In addition, the measure of weights for each disease [3-6]. (54 items) also is importance, but also depends with different factors mostly age, gender and region [7-13]. These could be considered as quantities score for each group by expert committee. In this study which was carried out for considering very more conventional items in 54 diseases and its relevant factors in order being sick to healthy. Perhaps expert committee may be added or omitted some item that could be considered which I have presented. Perhaps some items may be more relevant in some regions those could be considered too. Since 2013, I have published a few articles about health and measurements, and I've described the benefits of this measurement method to the world. ${ }^{14-15}$

\section{Conclusion:}

However, some health-related items always show not changing but the same items may be changed in terms of conditions and cause temporary or permanent illness, and thus change the health number. Therefore, the health number needs to be in line with the individual-general health and international standards of health, and everyone needs to work to upgrade them. I hope everyone can be informed of their health status and have a score for achieving their full health and if they do not know what problems they are and how they can overcome them. Therefore, the world needs to have some little look quantities at health as well as a qualitative look. My formula and easy calculation can be one of the solutions. My examples point to this fact. No one in the world has ever said it before.

\section{REFERENCES}

[1] Preamble to the Constitution of the World Health Organization as adopted by the International Health Conference, New York, and 19-22 June, 1946; signed on 22 July 1946 by the representatives of 61 States (Official Records of the World Health Organization, No. 2, p. 100) and entered into force on 7 April (1948). Available from https://www.who.int/governance/eb/who constitution en.pdf (1th Jul 2019).

[2] A Mehrabi Tavana. Mehdi Mehrabi Tavana, A Hypothesis: Introduce a Formula for Calculation of Human Health. Ann Med Health Sci Res. Vol 7:5 pp 46-50 (October 2017). Available from https://www.amhsr.org/articles/innovation-a-hypothesis-introduce-aformula-for-calculation-of-human-health-3819.html ( 1th Jul 2019).

[3] M Stouthard M, M ,Essink-Bot G Bonsel, J Barendregt \& P Kramers. Disability weights for diseases in the Netherlands. Rotterdam: Department of Public Health, Erasmus University. 1997. Available from https://dare.uva.nl/search?identifier=e7cbed27-7fab-4104-9b44$1657515747 \mathrm{c} 2$. (1th Jul 2019).

[4] S.Tretli. Height and weight in relation to breast cancer morbidity and mortality. A prospective study of 570,000 women in Norway. International Journal of Cancer vol 44: pp 23-30 (July 1989). Available from https://onlinelibrary.wiley.com/doi/abs/10.1002/ijc.2910440105 (1th Jul 2019).

[5] GM Tallis,, P Leppard \& TJ, O'Neill. The analysis of survival data from a central cancer registry with passive follow up. Statistics in Medicine vol 7:pp 483-490 (April 1988).

[6] A Tavani \& C, La Vecchia. Fruit and vegetable consumption and cancer risk in a Mediterranean population. American Journal of Clinical Nutrition vol 61(6 Suppl): pp1374S-1377S, (Jun1995). Available from https://www.ncbi.nlm.nih.gov/pubmed/7754990. DOI: 10.1093/ajcn/61.6.1374S. (1th Jul 2019).

[7] The global burden of disease: 2004 update. Geneva, World Health Organization, 2008.Available from https://www.who.int/healthinfo/global_burden disease/2004_report update/en/ (1th Jul 2019).

[8] CJL Murray, AD Lopez, RE, Black, CD, Mathers, K, Shibuya, M, Ezzati et al. Global burden of disease 2005: call for collaborators. Lancet, vol 370: pp 109-110. (Jul 2007). Available from https://www.ncbi.nlm.nih.gov/pubmed/9164317. PMID:9164317, DOI: 10.1016/S0140-6736(96)07495-8. (1th Jul 2019).

[9] G Danaei, CMM Lawes, S Vander Hoorn, CJL,Murray M Ezzati. Global and regional mortality from ischemic heart disease and stroke attributable to higher-than-optimal blood glucose concentration: comparative risk assessment. Lancet, vol 368: pp1651-1659. (Nov 2006). Available from https://www.hsph.harvard.edu/majidezzati/publications/ (1th Jul 2019).

[10] World Health Organization, UNICEF. Meeting the MDG drinkingwater and sanitation target: the urban and rural challenge of the decade. Geneva, World Health Organization and UNICEF Joint Monitoring Programme for Water Supply and Sanitation, 2006. Available from https://apps.who.int/iris/handle/10665/43488. (1th Jul 2019).

[11] Global status report on road safety: time for action. Geneva, World Health Organization, 2009.

[12] World report on road traffic injury prevention. Geneva, World Health Organization, 2004. Available from https://www.who.int/violence_injury_prevention/publications/road_tr affic/world_report/en/ (1th Jul 2019).

[13] S Lewington, G Whitlock, R Clarke, P Sherliker, J Emberson, J Halsey et al. Blood cholesterol and vascular mortality by age, sex, and blood pressure: a meta-analysis of individual data from 61 prospective studies with 55,000 vascular deaths. Lancet, vol 370: pp1829-1839.( Dec 2007). Available from https://www.ncbi.nlm.nih.gov/pubmed/18061058. (1th Jul 2019). PMID: 18061058 DOI: 10.1016/S0140-6736(07)61778-4

[14] A. M. Tavana, Does the health of individuals have a mathematical code? Int J Prev Med, vol 4: 849-851 (Jul 2013). Available from. PMID: 24049606 PMCID: PMC3775227 (1th Jul 2019).

[15] A Mehrabi Tavana. What are the Advantages of Considering a Formula for the Calculation of Human Health on both National and International Levels? Ann Med Health Sci Res.vol 7: 312-313 (September-October 2017).). Available from https://www.amhsr.org/articles/what-are-the-advantages-ofconsidering-a-formula-for-the-calculation-of-human-health-on-bothnational-and-international.pdf (1th Jul 2019). 


\section{ACKNOWLEDGMENT}

I would like to thank all those who have contributed to this research, especially Dr. Ahmad ali Noorbala and Dr. Ramadan Ali Ataee, and my son Dr. Mehdi Mehrabi Tavana. And I thank each and every one of my family members.

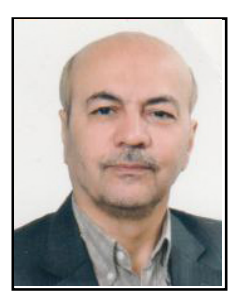

A. Mehrabi Tavana was born in Hamadan 1958 and got B.Sc. in Health from SBUMS, Tehran Iran, M.Sc. in Health Science TUMS, Tehran Iran, and Ph.D. in Medical Microbiology Manchester University. The author has experienced in the field of public health for many years and has written more than 100 papers in this filed. The author's major field of study is public health and how infectious diseases could be prevented. 